\title{
Mask-Wearing Increased After a Government Recommendation: A Natural Experiment in the U.S. During the COVID-19 Pandemic
}

\author{
Matthew H. Goldberg ${ }^{1 *}$, Abel Gustafson ${ }^{1}$, Edward W. Maibach ${ }^{2}$, Matthew T. Ballew ${ }^{1}$, \\ Parrish Bergquist ${ }^{1}$, John E. Kotcher ${ }^{2}$, Jennifer R. Marlon ${ }^{1}$, Seth A. Rosenthal ${ }^{1}$ and \\ Anthony Leiserowitz ${ }^{1}$ \\ ${ }^{1}$ Yale Program on Climate Change Communication, Yale University, New Haven, CT, United States, ${ }^{2}$ George Mason \\ University Center for Climate Change Communication, George Mason University, Fairfax, VA, United States
}

\section{OPEN ACCESS}

Edited by:

Mohan Jyoti Dutta,

Massey University, New Zealand

Reviewed by:

Peter Johannes Schulz,

University of Lugano, Switzerland

Shaunak Sastry,

University of Cincinnati, United States

Yuping Mao,

California State University, Long

Beach, United States

${ }^{*}$ Correspondence:

Matthew H. Goldberg

matthew.goldberg@yale.edu

Specialty section:

This article was submitted to

Health Communication,

a section of the journal

Frontiers in Communication

Received: 30 April 2020

Accepted: 02 June 2020

Published: 17 June 2020

Citation:

Goldberg MH, Gustafson A, Maibach EW, Ballew MT, Bergquist $P$, Kotcher JE, Marlon JR, Rosenthal SA

and Leiserowitz A (2020)

Mask-Wearing Increased After a

Government Recommendation: A Natural Experiment in the U.S. During

the COVID-19 Pandemic

Front. Commun. 5:44.

doi: 10.3389/fcomm.2020.00044
On April 3 2020, the U.S. Centers for Disease Control and Prevention (CDC) recommended that all Americans wear face masks to prevent the spread of COVID-19. The announcement came during the fielding of a large, nationally-representative survey ( $N=3,933$ ) of Americans' COVID-19-related knowledge, attitudes, and behaviors, providing an opportunity to measure the impact of the CDC's recommendation on public reported mask wearing and buying behavior. The study found significant increases in reported mask wearing ( +12 percentage points) and mask buying ( +7 points). These findings indicate the speed with which government recommendations can affect the adoption of protective behaviors by the public. The results demonstrate the importance of national leadership and communication during a public health crisis.

Keywords: COVID-19, government recommendation, CDC - Centers for Disease Control and Prevention, mask, preventive behavior

\section{INTRODUCTION}

The current coronavirus pandemic (COVID-19) has caused dramatic increases in morbidity, mortality, and economic strain. Because a vaccine is not currently available, individual preventive behaviors are the front line of defense against the spread of the disease. The recommendations of experts are a key source of information for the public (Covello et al., 2012; Peters, 2014), and their recommendations can be effective in correcting misinformation (Vraga and Bode, 2017). Thus, understanding how the public responds to the recommendations of experts and government sources is crucial for guiding ongoing public communication efforts. Further, the effects of government agencies' recommendations-and the agencies' corresponding credibility-illustrate the importance of national leadership and clear guidance in times of crisis. In this paper, we examine how a recommendation by the U.S. Centers for Disease Control and Prevention (CDC) that the public should wear protective masks influenced Americans' mask-wearing behavior.

Prior research has shown that people say they would be willing to enact a wide range of behaviors to prevent the spread of an influenza pandemic if, hypothetically, public health officials asked them to. For example, Blendon et al. (2008) found that most Americans said they would avoid air travel (93\%), avoid public events (92\%), or postpone personal events such as parties or funerals (79\%) if public health officials recommended it. Despite this evidence that Americans are willing to follow official recommendations, it is unclear how actual behaviors differ from hypothetical scenarios, how large these effects are, and how quickly they can take hold. 
In this study, we measure self-reported mask wearing during a pandemic before and after the U.S. Centers for Disease Control and Prevention (CDC) recommended that all Americans start wearing face masks to help prevent contracting or spreading COVID-19. We use the timing of the recommendation to assess a natural experiment of whether and how the American public responded to a preventive recommendation by the U.S. government. Our findings advance understanding of behavioral responses among the public to a government health communication in times of crisis, and provide a valid estimate of the size of the effect observed in the real world.

On the evening of April 3, 2020, the U.S. Centers for Disease Control and Prevention (CDC) announced new recommendations that all Americans wear face masks. President Trump mentioned this recommendation in his nationally televised public address on the evening of April 3, although some news outlets reported on the probable recommendation beforehand. News outlets began reporting on the actual recommendation late that same evening, and discussion of it in news and social media expanded quickly throughout the day on April 4.

The CDC recommendation to wear masks came after a series of mixed messages from different sources in the preceding weeks. For instance, the U.S. Surgeon General tweeted that masks are not effective, and the World Health Organization decided not to recommend that everyone wear masks (Jingnan, 2020; Sample, 2020). The CDC recommendation seemed to provide clarity and a consistent narrative, based on new knowledge that the virus can spread via asymptomatic individuals, and that the risk of infection could be reduced if wearing masks or other face coverings were widely adopted (Jingnan, 2020).

This breaking news and ensuing coverage occurred while we were fielding a large, nationally-representative survey that measured COVID-19-related knowledge, attitudes, and behaviors, providing an opportunity to test the effect of the government's recommendations as a natural experiment. That is, how did reported mask buying and mask wearing change from the days before the CDC's official recommendation to the days immediately after?

\section{METHOD}

\section{Participants}

A national sample of respondents in the United States $(N$ $=4,493$ ) was recruited by Climate Nexus Polling (April 37, 2020), which utilized several market research panels in the U.S. and recruited respondents using stratified sampling methods. Compensation for participants depended on the specific market research panel and respondents' preferences (e.g., cash, gift cards, reward points). Quotas were set to match census parameters for sex, race, age, education, income, and geographic region, and sampling weights were used to account for any small deviations from census parameters. Weighted and unweighted sample demographic breakdowns are available in Supplementary Table 1. This research was approved by the
Yale University and George Mason University institutional review boards.

Criteria for handling data exclusions were set before analysis. A total of 560 respondents were not included in analyses because they dropped out of the study soon after starting, did not reach the demographic section of the survey, were not living in the United States, were under 18 years old, or completed the survey in $<28 \%$ of the median response time. After dropping these respondents, the final sample of 3,933 was retained for the following analyses $\left(n_{\text {April3 }}=1,740, n_{\text {April4 }}=1,745, n_{\text {April5 }}=292\right.$, $\left.n_{\text {April6 }}=154, n_{\text {April7 }}=2\right)$.

\section{Materials and Procedure}

Respondents were asked diverse questions, including questions about their media consumption, trust in various sources for information about COVID-19, personal values, political beliefs, and which disease-preventive actions they had taken as a result of the COVID-19 pandemic. To measure individuals' behaviors,

TABLE 1 | Sample characteristics and balance tests for differences across treatment groups.

\begin{tabular}{|c|c|c|c|}
\hline Variable & April 3-4 & April 5-7 & Sig. Diff.? \\
\hline$N$ & 3,485 & 448 & \\
\hline Age & $M=47$ & $M=46$ & $\mathrm{~N}$ \\
\hline Sex & & & $\mathrm{N}$ \\
\hline Male & $47 \%$ & $44 \%$ & \\
\hline Female & $53 \%$ & $56 \%$ & \\
\hline Education & & & $\mathrm{N}$ \\
\hline Less than high school & $3 \%$ & $5 \%$ & \\
\hline High school & $27 \%$ & $24 \%$ & \\
\hline Some college & $41 \%$ & $41 \%$ & \\
\hline College degree or higher & $29 \%$ & $31 \%$ & \\
\hline Income & & & Y \\
\hline$<\$ 50,000$ & $50 \%$ & $57 \%$ & \\
\hline$\$ 50,000-\$ 99,999$ & $34 \%$ & $30 \%$ & \\
\hline$\$ 100,000$ or more & $16 \%$ & $14 \%$ & \\
\hline Race/Ethnicity* & & & Y \\
\hline Person of color & $29 \%$ & $84 \%$ & \\
\hline White, non-Hispanic & $72 \%$ & $16 \%$ & \\
\hline Political Party & & & Y \\
\hline Republican & $39 \%$ & $23 \%$ & \\
\hline Democrat & $43 \%$ & $54 \%$ & \\
\hline Independent & $11 \%$ & $12 \%$ & \\
\hline No party & $6 \%$ & $9 \%$ & \\
\hline Region & & & Y \\
\hline Northeast & $20 \%$ & $13 \%$ & \\
\hline Midwest & $22 \%$ & $14 \%$ & \\
\hline West & $19 \%$ & $28 \%$ & \\
\hline South & $39 \%$ & $45 \%$ & \\
\hline Coronavirus harm timing ${ }^{*}$ & & & $\mathrm{~N}$ \\
\hline They are being harmed right now & $55 \%$ & $53 \%$ & \\
\hline Other & $46 \%$ & $47 \%$ & \\
\hline
\end{tabular}

*Variable was dichotomized because of low sample sizes for select response options. Sig. Diff, Significant difference; Y, Yes; N, No. Tests of significance were assessed at a $p<0.05$ alpha level. 
we asked "Which, if any, of the following actions have you taken because of the spread of the coronavirus?" (Yes = 1; No, I prefer not to $=0 ;$ No, I'm not able to $=0$; Don't know = missing; Does not apply to $m e=$ missing). Respondents reported whether or not they had performed each of 27 behaviors, 23 of which were chosen specifically because they help prevent the contraction and spread of COVID-19 (e.g., bought protective masks; worn a mask in public to help protect yourself or others from getting sick; more frequently washed your hands with soap and water; kept at least 6 feet away from other people outside your home; stayed home instead of going to work, school, or gatherings). All survey questions used in this study and corresponding variable codes are available in the Supplementary Information. The Supplementary Information also details the primary results of this study when using alternate coding and adjustment methods.

To increase confidence that the CDC recommendation was responsible for any observed change in mask-related behavior, it is necessary to establish that (a) mask-related behavior was not already increasing at a similar rate prior to the CDC recommendation, and also that (b) other preventive behaviors did not similarly increase at the same time. To accomplish the first, we assessed day-to-day change from before the recommendation to immediately after (April 3rd to April 4th), which can reveal whether observed changes in the following days (once the information had been widely disseminated to, and consumed by, the public) is atypical. To accomplish the second, we assessed the degree to which other COVID-19 preventive behaviors changed across the same time period.

We used regression analyses to compare the likelihood of mask-related preventive behaviors between April 3 and April 4 (i.e., change from the day before to immediately after the recommendation; $n=3,485)$. Similarly, we used regression analyses to compare the likelihood of taking/engaging in maskrelated preventive behaviors between this initial time period (April 3-4) and the days that followed (April 5-7; $n=448$ ).
To rule out differences in sample characteristics based on when respondents completed the survey, as well as differences in the spatial trajectory of the spread of the virus, we tested whether the samples significantly differed in demographic breakdown (sex, age, race/ethnicity, income, education), political party, geographic region, and whether respondents believed their community was currently being harmed by COVID-19 (see Results section). Data and analysis code are available on our Open Science Framework project page at https://osf.io/8th6x/.

\section{RESULTS}

First, we examined the unadjusted mean differences in reported mask buying and mask wearing. From April 3 to 4, there was no significant change in reported mask buying $(+2$ percentage points, $95 \% \mathrm{CI}[-2,5])$ or mask wearing (+2 pts, 95\% $\mathrm{CI}[-2,5])$. However, once the CDC recommendation had been disseminated for at least one full day (i.e., comparing the April 3-4 period to the April 5-7 period), there were large increases in reported mask wearing (+21pts, 95\% CI[16, 27]; 48 to 69\%) and mask buying (+16 pts, $95 \%$ CI $[11,21]$; 43 to $59 \%$ ). However, tests for sample differences between time periods show that the samples significantly differed by income, race/ethnicity, political party, and geographic region (Table 1). Thus, to get a more accurate estimate of effect size, we controlled for these differences in all of the following analyses. Importantly, there was no difference across time periods in respondents' perceptions of whether their community was currently being harmed by COVID-19. This gave us more confidence in the internal validity of comparing results from the different time periods (April 3-4 vs. April 5-7).

Results controlling for income, race/ethnicity, political party, and geographic region showed there was no significant change from April 3 to 4 in reported mask buying ( +2 pts, $95 \%$ CI[-2, 5]) or mask wearing (0 pts, 95\% CI $[-3,4])$. Again, however, once the CDC recommendation had been disseminated for at least 1 day, there were large increases in reported mask wearing $(+12$

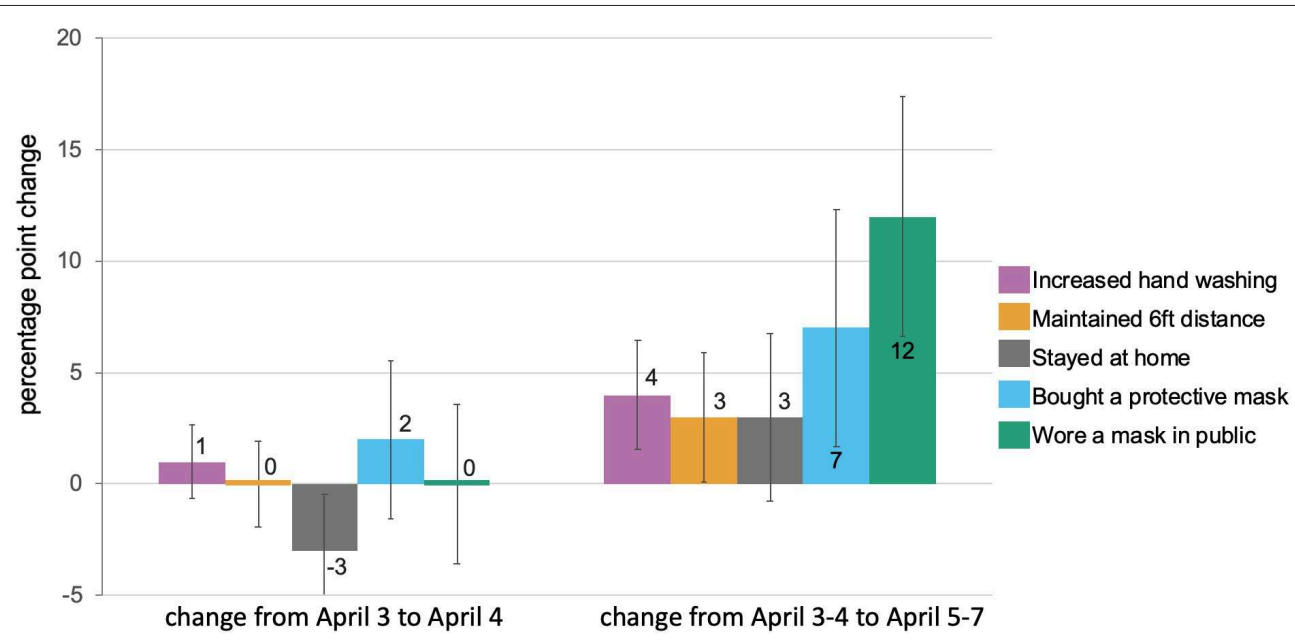

FIGURE 1 | Changes in Americans' COVID-19 preventive behaviors before and after the CDC recommendation that all Americans wear masks. Error bars represent $95 \%$ confidence intervals. 
pts, $95 \% \mathrm{CI}[7,18] ; 49$ to $61 \%)$ and mask buying (+7 pts, $95 \%$ $\mathrm{CI}[2,13] ; 44$ to $51 \%)^{1}$.

$Z$-tests indicated that the change between the April 3-4 period and the April 5-7 period was significantly larger for mask wearing $(Z=3.58, p<0.001)$ and marginally larger for mask buying $(Z$ $=1.67, p=0.096$ ), compared to the changes in those behaviors between April 3 and April 4. Further, the increase in mask wearing after the CDC recommendation was significantly larger than the increase in 18 of the 22 other preventive behaviors we measured, and descriptively larger than the increase in the other four (see Figure 1; also see Supplementary Table 3).

As an additional robustness check, we examined whether other behaviors increased more rapidly over the same time period. For example, were people simply paying closer attention to the news about COVID-19 in the days following the CDC recommendation to wear masks? Results show that, if anything, there was a significant decrease in attention to the news from the day before to the day after the recommendation $(b=-0.06,95 \%$ $\mathrm{CI}[-0.10,-0.02])$, but no difference in news attention in the days that followed $(b=0.03,95 \% \mathrm{CI}[-0.03,0.09])$.

Next, we examined whether the increases in mask wearing and mask buying behaviors were larger for people with more trust in various sources of information, and also whether the increases were larger for people who were paying closer attention to news about COVID-19. The increase in mask wearing was significantly larger for people who reported more trust in infectious disease experts $(b=0.07, S E=0.03), p=0.023$, $95 \% \mathrm{CI}[0.01,0.14]$ and marginally larger for people with more trust in the CDC $(b=0.06, S E=0.03), p=0.068,95 \%$ $\mathrm{CI}[-0.00,0.12]$, but did not vary based on people's reported levels of trust in President Trump $(b=0.00, S E=0.02), p=$ $0.946,95 \% \mathrm{CI}[-0.04,0.05]$, or their attention to news about COVID-19 $(b=0.01, S E=0.04), p=0.758,95 \% \mathrm{CI}[-0.06$, 0.09]. Among people who reported that they strongly trust infectious disease experts $(n=2,042)$, for example, there was a 17 percentage point increase in mask wearing in the days following the official recommendation (95\% CI [10, 25]). In contrast, increases in mask buying were mostly consistent regardless of people's trust in infectious disease experts $(b=0.04, S E=$ $0.03), p=0.248,95 \% \mathrm{CI}[-0.03,0.10]$, the CDC $(b=0.04, S E$ $=0.03), p=0.166,95 \% \mathrm{CI}[-0.02,0.10]$, or President Trump $(b=-0.02, S E=0.02), p=0.499,95 \% \mathrm{CI}[-0.06,0.03]$, and was also consistent across different levels of attention to news about COVID-19 ( $b=-0.06, S E .04), p=0.123,95 \%$ $\mathrm{CI}[-0.14,0.02]$.

It is important to note that using the timing of the CDC recommendation to separate the two time periods we compare is imperfect. That is, after the CDC recommendation, people likely did not hear about it simultaneously or from the same sources. To provide additional context and corroborating evidence, we

\footnotetext{
${ }^{1}$ To ensure sample differences in the different time periods were appropriately balanced, and that corresponding results did not depend on the method used, we reran analyses using entropy balancing (Hainmueller, 2012). Results were nearly identical for both mask wearing ( +13 pts, 95\% CI[7, 18]), mask buying $(+8$ pts, 95\% CI[2, 13]), and other preventive behaviors (see Supplementary Table 6). For ease of communication and likely higher familiarity with regression-based covariate adjustment among readers, we use multiple regression in the main text.
}

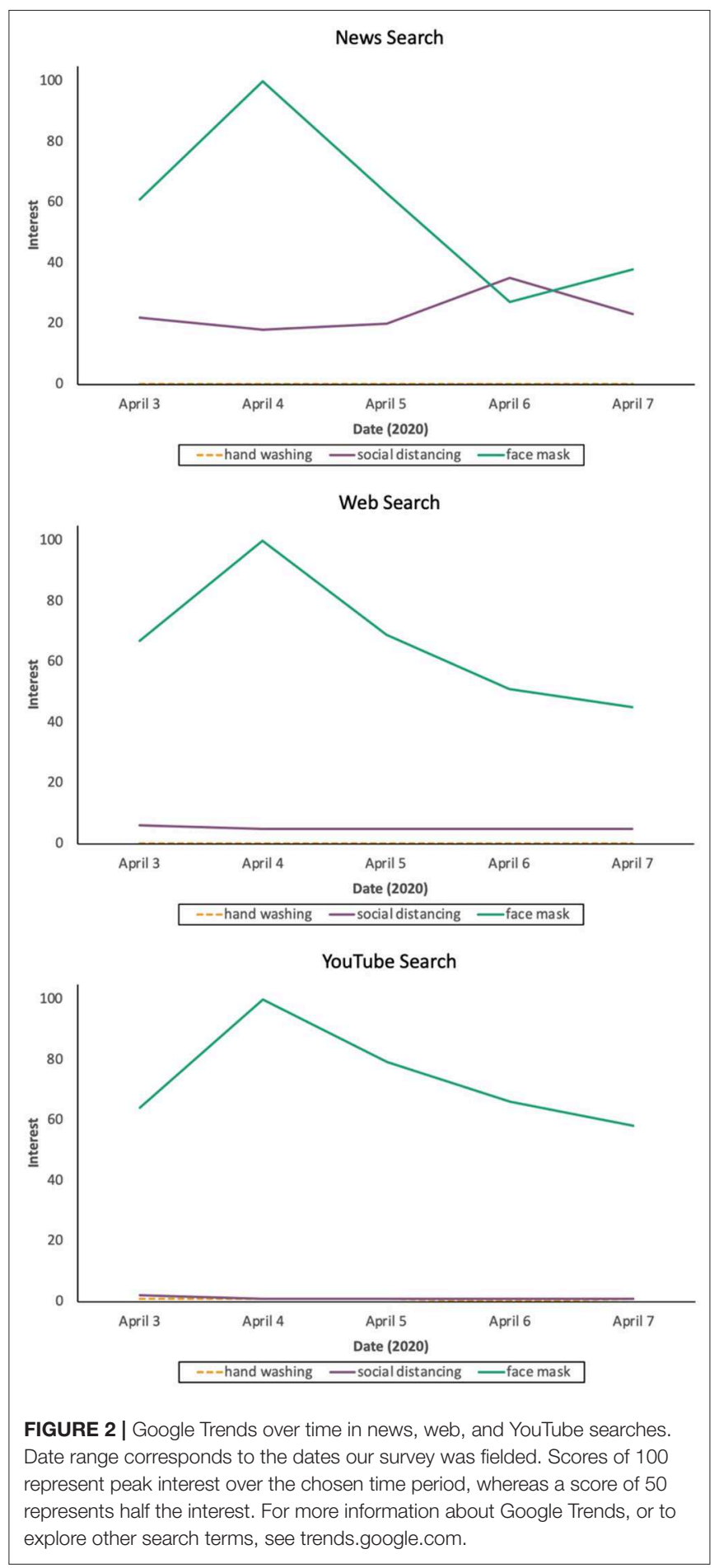

examined Google Trends news, web, and YouTube searches over the same time period our survey was in the field.

Results showed that news, web, and YouTube searches for "face mask" all peaked on April 4-the day after the CDC recommendation. Over this same time period, searches for "social distancing," "hand washing," and other preventive behaviors did not show the same spike in interest, further suggesting that the $\mathrm{CDC}$ recommendation and the ensuing news 
coverage increased interest and consumption of information related to masks specifically, rather than an increase in searches about preventive behaviors in general (Figure 2).

\section{DISCUSSION}

In our national survey, which straddled the release of the CDC recommendation to wear masks, we observed a large increase (12 percentage points) in reported mask wearing, which, when extrapolated to the U.S. population, represents many millions of American adults. The effect of the CDC recommendation was greater among those who have more trust in the CDC and infectious disease experts as sources of information about COVID-19. Consistent with previous research (Druckman, 2001), these results suggest that efforts made by government agencies and scientists to cultivate rapport with and trust from the public improve the likelihood that their recommendations are heeded in times of crisis.

A descriptively smaller but significant increase ( 7 percentage points) was also observed in mask buying over the same time period. Given that people who have masks can immediately start wearing them, and others can make their own (or use alternatives such as scarves or other clothing), this smaller effect may reflect the increased difficulty in buying masks, which takes more time and effort (and was impossible in some cases).

This study has limitations that should be noted. First, the political and social landscape around COVID-19 is changing rapidly and showing signs of increasing partisan division (van der Linden et al., 2020). Thus, although the current study demonstrates the large and immediate impact government recommendations can have, other important factors, such as social norms, changes in risk perceptions, and cues from elites, including the media (Dryhurst et al., 2020; Goldberg et al., 2020; Van Bavel et al., 2020), are likely to have increasing influence over mask-wearing behavior.

An additional limitation is that, although we found larger increases in mask wearing among people who reported stronger trust in infectious disease experts and the $\mathrm{CDC}$, any variables strongly correlated with trust in these entities could theoretically moderate the results as well. For example, greater trust in these entities can reflect differences in political partisanship or socioeconomic status. Despite this limitation, the current results are consistent with experimental research showing that credible sources are more influential than sources that are not as credible in the relevant topic area (Druckman, 2001).

Further, given the quickly-changing social and political landscape and trajectory of the pandemic, it is not known how long this increase in mask wearing will last and whether repeated recommendations will have the same effect. Previous research on other topics suggests that the durability of similar messaging effects depends on how strongly people formed their initial attitude toward the message (Chong and Druckman, 2010), whether the recommendation is repeated (Carnahan et al., 2020), and whether people consume information that bolsters or competes with the recommendation-via conversation with others or via additional media consumption (Druckman and Nelson, 2003; Chong and Druckman, 2013; Goldberg et al., 2019; Dryhurst et al., 2020).
Finally, our measure of mask wearing (and other preventive behaviors) is another limitation. First, we rely on self-reports. Thus, our measure is susceptible to bias depending on whether respondents see particular preventive behaviors as (un)favorable to their image. It is worth noting that respondents were assured their responses were anonymous, but even anonymous responses may be subject to social desirability bias. Further, because we did not anticipate the timing of the government recommendation and widespread advice from experts that everyone wear a maskeven if just a cloth face covering (e.g., scarf, bandana, homemade mask) -we did not specifically ask respondents about cloth face coverings. We therefore do not know if respondents considered cloth face coverings or home-made masks when reporting whether or not they bought or wore a mask.

Despite these limitations, our findings provide evidence that official recommendations in times of crisis can have large and immediate effects on the health behaviors of the public and provide a robust estimate of the size of those effects in a real crisis. These findings provide a rare glimpse into the speed with which recommendations from trusted officials can begin to affect the health behaviors of the American public, and point to the importance of consistent, credible national leadership in times of crisis.

\section{DATA AVAILABILITY STATEMENT}

The datasets presented in this study can be found in online repositories. The names of the repository/repositories and accession number(s) can be found below: Data are available on our Open Science Framework project page at: https://osf.io/8th6x.

\section{ETHICS STATEMENT}

The studies involving human participants were reviewed and approved by Yale University and George Mason University. The patients/participants provided their written informed consent to participate in this study.

\section{AUTHOR CONTRIBUTIONS}

All authors contributed to the survey design, which was led by MB. MG conceptualized the idea and analyzed the data. MG and AG drafted the first draft of the manuscript and all authors contributed edits and recommendations that improved the manuscript and analyses. All authors approved of the final version of the manuscript.

\section{FUNDING}

The research was funded by the Catalyst Fund at the Yale School of the Environment.

\section{SUPPLEMENTARY MATERIAL}

The Supplementary Material for this article can be found online at: https://www.frontiersin.org/articles/10.3389/fcomm. 2020.00044/full\#supplementary-material 


\section{REFERENCES}

Blendon, R. J., Koonin, L. M., Benson, J. M., Cetron, M. S., Pollard, W. E., Mitchell, E. W., et al. (2008). Public response to community mitigation measures for pandemic influenza. Emerg. Infect. Dis. 14, 778-786. doi: 10.3201/eid1405.071437

Carnahan, D., Bergan, D. E., and Lee, S. (2020). Do corrective effects last? Results from a longitudinal experiment on beliefs toward immigration in the US. Political Behav. 1-20. doi: 10.1007/s11109-020-09591-9

Chong, D., and Druckman, J. N. (2010). Dynamic public opinion: communication effects over time. Am. Political Sci. Rev. 104, 663-680. doi: $10.1017 /$ S0003055410000493

Chong, D., and Druckman, J. N. (2013). Counterframing effects. J. Politics 75, 1-16. doi: $10.1017 /$ S0022381612000837

Covello, V. T., McCallum, D. B., and Pavlova, M. T. (Eds.). (2012). Effective Risk Communication: The Role and Responsibility of Government and Nongovernment Organizations, Vol. 4. New York, NY: Springer Science \& Business Media.

Druckman, J. N. (2001). On the limits of framing effects: who can frame? J. Politics. 63, 1041-1066. doi: 10.1111/0022-3816.00100

Druckman, J. N., and Nelson, K. R. (2003). Framing and deliberation: How citizens' conversations limit elite influence. Am. J. Political Sci. 47, 729-745. doi: 10.1111/1540-5907.00051

Dryhurst, S., Schneider, C. R., Kerr, J., Freeman, A. L., Recchia, G., Van Der Bles, A. M., et al. (2020). Risk perceptions of COVID-19 around the world. J. Risk Res. 1-13. doi: 10.1080/13669877.2020.1758193

Goldberg, M., Gustafson, A., Maibach, E., van der Linden, S., Ballew, M. T., Bergquist, P., et al. (2020). Social norms motivate COVID-19 preventive behaviors. PsyArXiv [Preprint]. doi: 10.31234/osf.io/9whp4

Goldberg, M. H., van der Linden, S., Maibach, E., and Leiserowitz, A. (2019). Discussing global warming leads to greater acceptance of climate science. Proc. Natl. Acad. Sci. U.S.A. 116, 14804-14805. doi: 10.1073/pnas.1906589116

Hainmueller, J. (2012). Entropy balancing for causal effects: a multivariate reweighting method to produce balanced samples in observational studies. Political Anal. 20, 25-46. doi: 10.1093/pan/mpr025
Jingnan, H. (2020). Why There Are so Many Different Guidelines for Face Masks for the Public. National Public Radio. Available online at: https://www.npr.org/ sections/goatsandsoda/2020/04/10/829890635/why-there-so-many-differentguidelines-for-face-masks-for-the-public (accessed May 24, 2020).

Peters, H. P. (2014). "Scientists as public experts: Expectations and responsibilities," in Routledge Handbook of Public Communication of Science and Technology, eds M. Bucchi and B. Trench (New York, NY: Routledge), 86-98.

Sample, I. (2020). Face masks cannot stop healthy people getting Covid-19, says WHO. The Guardian. Available online at: https://www.theguardian.com/ world/2020/apr/07/face-masks-cannot-stop-healthy-people-getting-covid19-says-who (accessed May 24, 2020).

Van Bavel, J. J., Baicker, K., Boggio, P. S., Capraro, V., Cichocka, A., Cikara, M., et al. (2020). Using social and behavioural science to support COVID-19 pandemic response. Nat. Hum. Behav. 1-12. doi: 10.31234/osf.io/y38m9

van der Linden, S., Panagopoulos, C., and Kerr, J. (2020). Coronavirus: New Survey Shows How Republicans and Democrats are Responding Differently. The Conversation. Available online at: https://theconversation.com/coronavirusnew-survey-shows-how-republicans-and-democrats-are-respondingdifferently-138394 (accessed May 24, 2020).

Vraga, E. K., and Bode, L. (2017). Using expert sources to correct health misinformation in social media. Sci. Commun. 39, 621-645. doi: $10.1177 / 1075547017731776$

Conflict of Interest: The authors declare that the research was conducted in the absence of any commercial or financial relationships that could be construed as a potential conflict of interest.

Copyright (C) 2020 Goldberg, Gustafson, Maibach, Ballew, Bergquist, Kotcher, Marlon, Rosenthal and Leiserowitz. This is an open-access article distributed under the terms of the Creative Commons Attribution License (CC BY). The use, distribution or reproduction in other forums is permitted, provided the original author(s) and the copyright owner(s) are credited and that the original publication in this journal is cited, in accordance with accepted academic practice. No use, distribution or reproduction is permitted which does not comply with these terms. 